\title{
CURVAS DE REFERENCIA DEL ÍNDICE DE MASA CORPORAL PARA NIÑAS, NIÑOS Y ADOLESCENTES COSTARRICENSES
}

\author{
José Moncada Jiménez \\ Docente e investigador de la Escuela de Educación Física y \\ Deportes de la Universidad de Costa Rica \\ San José, Costa Rica
}

Recibido 12-VIII-2008 • Aceptado 18-IX-2008 • Corregido 9-VI-2009

Resumen: En el artículo se presentan curvas del indice de masa corporal para hombres $(n=5031) y$ mujeres $(n=4959)$ costarricenses entre 8 y 17 años. Se destacan los percentiles que definen desnutrición, peso normal, sobrepeso y obesidad.

Palabras clave: Curvas de referencia, adiposidad, niños y niñas, adolescentes.

\begin{abstract}
In the article, body mass index reference curves for Costa Rican men $(n=5031)$ and women $(n=4959)$ between 8 and 17 years are presented. The percentiles that define undernourishment, normal weight, overweight and obesity stand out.
\end{abstract}

Key words: Reference curves, adiposity, children, adolescents.
El control del peso y la estatura en la población es importante para determinar patrones de desnutrición, peso normal, sobrepeso y obesidad. Existen gráficos o curvas de crecimiento desarrolladas para poblaciones estadounidenses y británicas (Cole, Freeman y Preece, 1995; National Center for Health Statistics, 2000); sin embargo, se ha discutido extensamente de la idoneidad de su uso en poblaciones hispanas o latinoamericanas, como por ejemplo, la costarricense.

En esta nota técnica se presentan dos figuras en las que se detallan las curvas de referencia del índice de masa corporal (IMC) para niñas, niños y adolescentes costarricenses entre 8 y 17 años. El IMC es un indicador válido para estimar la cantidad de grasa que tienen las personas comunes, no atletas, y se obtiene al dividir el peso en kilogramos entre el cuadrado de la estatura en metros, según la fórmula: $\mathrm{IMC}=$ Peso $(\mathrm{kg}) /$ estatura $\left(\mathrm{m}^{2}\right)$. El IMC se basa en el concepto de que el peso de una persona debe ser proporcional a su estatura. En personas adultas, un IMC menor de 18,5 sugiere malnutrición, entre 
18 y 25 es normal, y valores superiores a 25 se relacionan con sobrepeso, obesidad, y la aparición de diabetes, hipertensión y enfermedades cardiovasculares (Brooks, Fahey, White y Baldwin, 2000).

Los datos utilizados para elaborar los gráficos forman parte de los resultados de una investigación realizada a nivel nacional publicada anteriormente (Fernández, González, Moncada, Pearson, Picado y Salas, 2001; Fernández, Pearson, Moncada, Salas y González, 1998; Fernández y Moncada, 2003). En síntesis, el estudio consistió en el establecimiento de normas nacionales de los componentes de salud física desarrollado por la Universidad de Costa Rica y el Ministerio de Educación Pública entre 1996 y 1998. La muestra representativa de la población costarricense de estudiantes entre los 8 y 17 años de ambos sexos fue en total de 9990 sujetos ( $n=5031$ hombres y $n$ $=4959$ mujeres).

En el estudio se midieron, entre otras variables, el peso y la estatura para obtener el IMC. El peso corporal se midió con el sujeto descalzo y con ropa ligera (pantalón corto y camiseta) sobre una báscula de plataforma marca Detecto ${ }^{\circledR}$, calibrada en $\mathrm{kg}(0,1 \mathrm{~kg}$ más cercano). Se realizó la lectura con el sujeto inmóvil, en posición erguida, la vista al frente y colocado en el centro de la balanza. Para medir la talla corporal en un tallímetro, calibrado en cm (0,1 cm más cercano), se colocó al sujeto descalzo y en posición erguida, de espalda al tallímetro. El estudiante debía tener los maléolos internos juntos y talones, glúteos, hombros y región posterior de la cabeza en contacto con el aparato. Posteriormente, con ambas manos se realizó una ligera tracción hacia arriba en la mandíbula, orientando la cabeza en un ángulo aproximado de $90^{\circ}$, con el sujeto viendo al frente. Todos estos procedimientos han sido previamente publicados (Fernández et al., 2001).

Para elaborar las curvas, se calcularon los percentiles para el IMC por separado para hombres y mujeres por medio del método LMS (Cole, 1990; Cole y Green, 1992). Con este método se pueden resumir los datos en términos de tres curvas suavizadas específicas para cada edad por medio de los parámetros L (lambda, $\lambda$ ), M (mu, $\mu$ ), y S (sigma $\sigma$, Las curvas de $\mu$ y $\sigma$ corresponden a la mediana y el coeficiente de variación del IMC en cada edad; mientras que la curva $\lambda$ permite corregir el sesgo en la distribución del IMC dependiente de la edad. Para la elaboración de las curvas se utilizó el programa estadístico LMS (versión 1,25) y la supervisión del Dr. David McCarthy (Department of Health and Human Sciences, London Metropolitan University, London), quien junto con su grupo de investigación han desarrollado curvas de referencia en otras poblaciones (McCarthy, Cole, Fry, Jebb y Prentice, 2006).

Se estimaron siete curvas (percentiles): 2, 9, 25, 50, 75, 91 y 98. Estas estuvieron separadas entre sí 0,66 desviaciones estándares. Para definir puntajes de corte del IMC que fueran clínica y epidemiológicamente consistentes con los reportados por la International Obesity Task Force (IOTF) y por Fernández y Moncada (2003), se obtuvieron los percentiles 85 y 95 para definir sobrepeso y obesidad, respectivamente. El percentil 50 indica el peso normal para la estatura. Se seleccionó el percentil 2 para definir el límite superior para una persona con bajo peso para su estatura; es decir, desnutrida o en peligro de desnutrición.

A continuación se presentan los gráficos para hombres y mujeres costarricenses entre 8 y 17 años. Se espera que con el uso de estos gráficos, el cuerpo docente de escuelas y colegios pueda cuantificar si existen niveles de riesgo que puedan afectar la salud de sus estudiantes. As su vez, se espera que se puedan llevar a cabo acciones concretas como la consejería nutricional y el ejercicio físico sistemático y supervisado que permita mejorar la calidad de vida de las niñas, niños y adolescentes a su cargo. 
Figura 1

Curvas de percentiles para el IMC $\left(\mathrm{kg} / \mathrm{m}^{2}\right)$ para hombres costarricenses entre 8 y 17 años $(\mathrm{n}=5023)$. Los datos fueron suavizados por medio del método LMS. Los números del lado derecho representan los percentiles

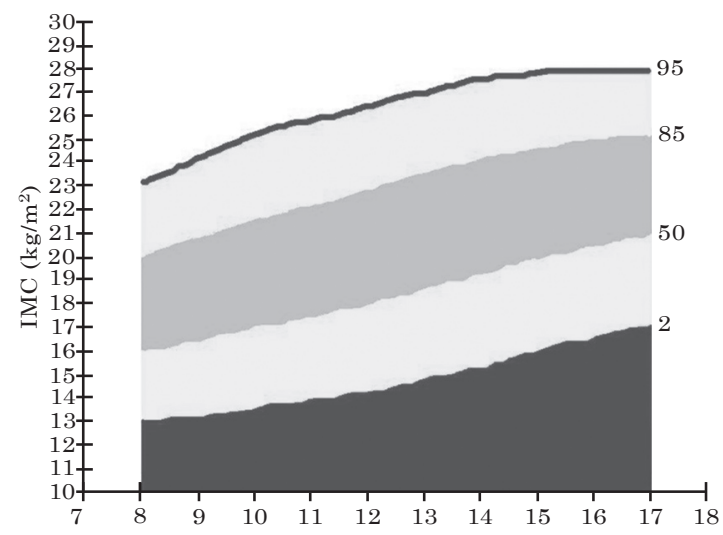

Edad

\section{Referencias bibliográficas}

Brooks, G. A., Fahey, T. D., White, T. P. y Baldwin, K. M. (2000). Exercise physiology: Human bioenergetics and its applications [Fisiología del ejercicio: Bioenergética humana y sus aplicaciones] ( $3^{r d}$ ed.). Mountain View, CA: Mayfield Publishing Co.

Cole, T. J. y Green, P. J. (1992). Smoothing reference centile curves: The LMS method and penalized likelihood [Suavizado de curvas de centiles de referencia]. Statistics in Medicine, 11, 1305-1319.

Cole, T. J. (1990). The LMS method for constructing normalized growth standards [El método LMS para la construcción de estándares normalizados de crecimiento]. European Journal of Clinical Nutrition, 44(1), 45-60.

Cole, T. J., Freeman, J. V. y Preece, M. A. (1995). Body mass index reference curves for the UK, 1990 [Curvas de
Figura 2

Curvas de percentiles para el IMC $\left(\mathrm{kg} / \mathrm{m}^{2}\right)$ para mujeres costarricenses entre 8 y 17 años $(\mathrm{n}=4950)$. Los datos fueron suavizados por medio del método LMS. Los números del lado derecho representan los percentiles

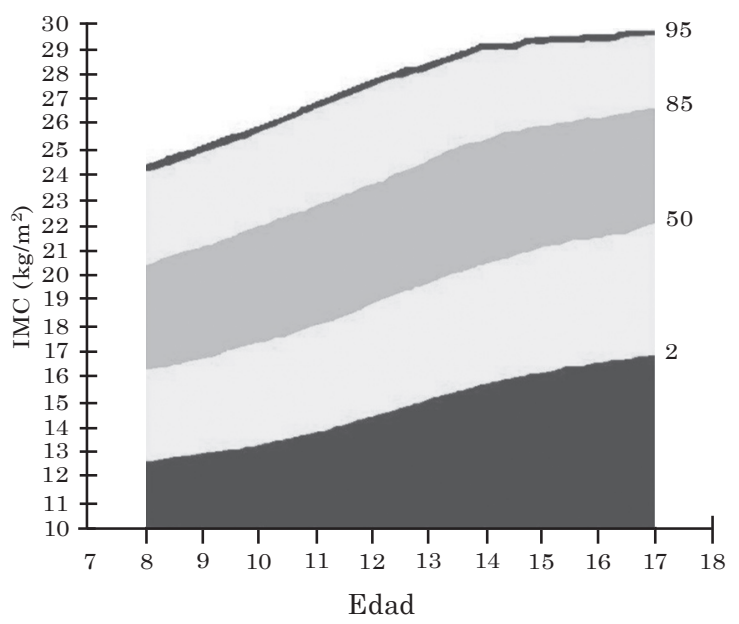

referencia de índice de masa corporal para el Reino Unido de 1990]. Archives of Disease in Children, 73, 25-29.

Fernández, A., González, C., Moncada, J., Pearson, G., Picado, M. y Salas, R. (2001). Normas nacionales. Componentes de salud física: Estudiantes costarricenses entre 8 y 17 años. San José, Costa Rica: Editorial Baula.

Fernández, A., Pearson, G., Moncada, J., Salas, R. y González, C. (1998). Evaluación de la salud física de estudiantes de la Educación General Básica. Programa de Medición y Evaluación Educativa. San José Costa Rica: Universidad de Costa RicaMinisterio de Educación Pública.

Fernández, A. y Moncada, J. (2003). Obesidad y sobrepeso en la población estudiantil costarricense entre los 8 y 17 años. Revista Costarricense de Ciencias Médicas, 24(3, 4), 95-113. 
McCarthy, H. D., Cole, T. J., Fry, T., Jebb, S. A. \& Prentice, A. M. (2006). Body fat reference curves for children [Curvas de referencia de grasa corporal para niños y niñas]. International Journal of Obesity, 30, 598-602.
National Center for Health Statistics. (2000). Pediatric Growth Charts [Tablas de crecimiento pediátricas]. Consultad0 el 07 de enero del 2008 de http://www.cdc.gov/ growthcharts. 